\title{
Apparent self-accelerating alternating assembly of semiconductor nanoparticles and polymers
}

ARTICLE in APPLIED PHYSICS LETTERS · JULY 2015

Impact Factor: $3.52 \cdot$ DOI: 10.1063/1.4927403

DOWNLOADS

10
VIEWS

34

3 AUTHORS, INCLUDING:

\section{Robert Horvath}

Institute of Technical Physics and Materials S.

75 PUBLICATIONS 860 CITATIONS

SEE PROFILE
Jeremy Ramsden

Collegium Basilea (Institute of Advanced Stu...

215 PUBLICATIONS $\quad 4,888$ CITATIONS

SEE PROFILE 


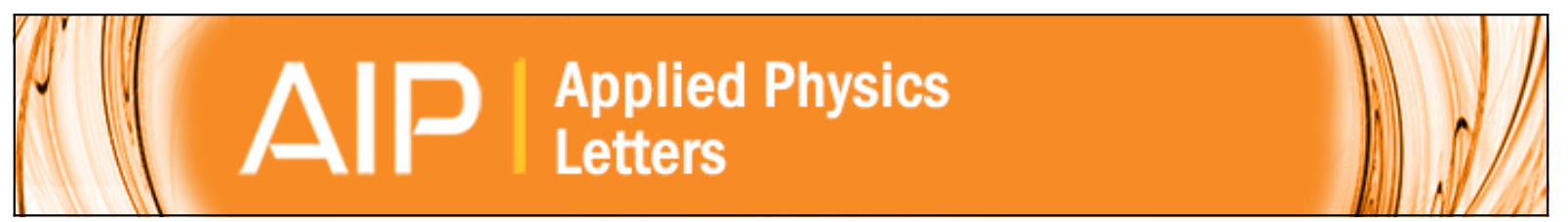

\section{Apparent self-accelerating alternating assembly of semiconductor nanoparticles and polymers}

R. Horvath, H. C. Gardner, and J. J. Ramsden

Citation: Applied Physics Letters 107, 041604 (2015); doi: 10.1063/1.4927403

View online: http://dx.doi.org/10.1063/1.4927403

View Table of Contents: http://scitation.aip.org/content/aip/journal/apl/107/4?ver=pdfcov

Published by the AIP Publishing

\section{Articles you may be interested in}

Exciton-plasmon coupling mediated photorefractivity in gold-nanoparticle- and quantum-dot-dispersed polymers Appl. Phys. Lett. 102, 251115 (2013); 10.1063/1.4812720

Role of SnO2 as a filler in (PEO)50AgCF3SO3 nanocomposite polymer electrolyte system AIP Conf. Proc. 1447, 399 (2012); 10.1063/1.4710048

Enhanced photorefractive effect in liquid crystal structures co-doped with semiconductor quantum dots and metallic nanoparticles

Appl. Phys. Lett. 99, 191109 (2011); 10.1063/1.3659485

Size effect of cubic ZrO2 nanoparticles on ionic conductivity of polyethylene oxide-based composite J. Appl. Phys. 110, 043707 (2011); 10.1063/1.3622671

Dispersion of $\mathrm{Cd} X(\mathrm{X}=\mathrm{Se}, \mathrm{Te})$ nanoparticles in $\mathrm{P} 3 \mathrm{HT}$ conjugated polymer

J. Renewable Sustainable Energy 1, 023107 (2009); 10.1063/1.3101815

Frustrated by

old technology?

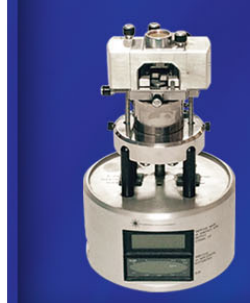

Is your AFM dead

and can't be repaired?

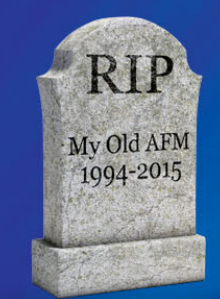

Sick of bad customer support?

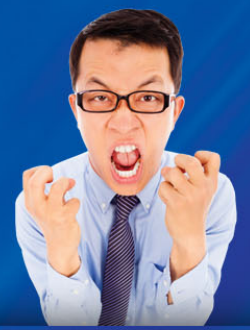

It is time to upgrade your AFM

Minimum $\$ 20,000$ trade-in discount for purchases before August 31st

Asylum Research is today's technology leader in AFM 


\title{
Apparent self-accelerating alternating assembly of semiconductor nanoparticles and polymers
}

\author{
R. Horvath, ${ }^{1,2}$ H. C. Gardner, ${ }^{2,3}$ and J. J. Ramsden ${ }^{2,4, a)}$ \\ ${ }^{1}$ Nanobiosensorics Laboratory, MTA-EK-MFA, Konkoly Thege út 29-33, 1121 Budapest, Hungary \\ ${ }^{2}$ Department of Materials, Cranfield University, MK43 OAL Bedfordshire, United Kingdom \\ ${ }^{3}$ Biomedical Engineering and Nanotechnology, Nanyang Polytechnic, 180 Ang Mo Kio Ave 8, Singapore \\ 569830 \\ ${ }^{4}$ Clore Laboratory, The University of Buckingham, Buckingham MK18 1EG, United Kingdom
}

(Received 6 May 2015; accepted 7 July 2015; published online 27 July 2015)

\begin{abstract}
Alternating polyelectrolyte deposition is a promising route to the low-cost fabrication of electroluminescent devices based on semiconductor nanoparticles, but optimization and exploitation demand a deeper understanding of the fabrication mechanism, which has not hitherto been scrutinized in detail. Nanoparticle-polymer composites were assembled by repeated alternate exposures of a substrate to polyanionic thioglycolate-coated CdTe nanoparticles and the organic polycation polydiallyldimethylammonium while monitoring the process kinetics in situ using optical waveguide lightmode spectroscopy, which enabled detailed structural information to be obtained with good time resolution. This complements the previously reported device characterization. Two hitherto unnoticed features were observed: (i) apparently spontaneous acceleration of addition of semiconductor nanoparticles after a certain quantity has already been deposited and (ii) during subsequent exposure to the organic polycation, an appreciable proportion of the immediately previously deposited nanoparticles is removed. Analysis of the evolution of the optogeometrical parameters of the assembly revealed that during the initial slow addition the nanoparticles enter nanopores in the immediately previously deposited polymer. The deposition régime then switches abruptly to the formation of an adlayer of the nanoparticles. These are initially deposited in considerable excess, which is removable by simple dilution of the system. Further nanoparticle removal takes place during the following phase of polycation deposition via a process of particle scavenging by the polycation molecules. Changes in film refractive index during these various processes show that the predominantly columnar (rather than laminar) molecular arrangement established for polyelectrolyte-only films is maintained in the hybrid polymer-particle films, although the filling of the polyelectrolyte pores makes the film more isotropic. (C) 2015 AIP Publishing LLC.
\end{abstract}

[http://dx.doi.org/10.1063/1.4927403]

Recently, functioning nanoparticle-based electroluminescent devices were assembled using alternating polyelectrolyte deposition (APED). ${ }^{2}$ Although in that work, the utility of APED (also loosely known as "layer-by-layer" (LbL) deposition) was amply demonstrated, the evolution of the structure of the devices during their formation was not investigated. In order to permit rational optimization of the assembly process, we have investigated it in situ using highresolution optical waveguide lightmode spectroscopy (OWLS).

APED enables a pair of oppositely electrostatically charged polyelectrolytes to be deposited on a substrate by exposing it alternatingly to solutions of the polyelectrolytes. The key feature enabling the deposition to continue indefinitely is that upon each exposure the charge is overcompensated. ${ }^{7}$ In the pioneering demonstration of the phenomenon, ${ }^{11}$ colloidal particles were used as the polyelectrolyte. The technique seems to have remained a laboratory curiosity for many years after its invention. Later, it attracted broader interest, organic polymeric polyelectrolytes were used, ${ }^{5,26}$ which became the "classic" process, ${ }^{4}$ with numerous applications

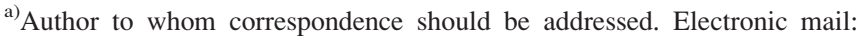
jeremy.ramsden@buckingham.ac.uk
}

being investigated, ranging from papermaking ${ }^{34}$ to biomedicine. ${ }^{3}$ An obvious development was to use nano-objects (colloidal particles, nanorods, and nanoplatelets) as one polyeletrolyte and an organic polymer as the other (e.g., Refs. $14,17,19$, and 33). It has not always been possible to observe the buildup of the film in any detail but where this has been done a monotonic increase in the amount of material deposited has been observed (e.g., Refs. 13, 26, and 31) - this can be considered to be the "classic" behaviour (when both polyelectrolytes are nano-objects then the behavior is typically more complicated $^{11,21}$ ).

In the course of the previous work, ${ }^{2}$ it became clear that in order to progress further with the technology (which should enable such devices to be fabricated at far lower cost than using conventional semiconductor processing technology - note that working photovoltaic devices based on this principle have been fabricated from $\mathrm{CdSe}$, albeit with low conversion efficiencies ${ }^{19}$ - and enable the emission spectrum to be defined simply by changing the nanoparticle size) deeper knowledge of the assembly process is needed to understand the controlling parameters. To achieve this, OWLS, whereby the nanoparticles are assembled with the help of a polyelectrolyte on a planar optical waveguide acting as the substrate, is useful because the optogeometric 
parameters of the assembled layer can be precisely determined in situ during the assembly process. ${ }^{21,24}$

Unexpectedly, we observed a striking departure from the "classic" behavior of a monotonically increasing deposit: in each cycle, after about $15 \%$ of the deposit had been achieved, the rate of deposition dramatically increased without any external intervention. We further observed that a considerable fraction was removed during exposure to the organic polymer polyelectrolyte in the next stage of the deposition cycle.

CdTe nanoparticles were a kind gift from Gaponik. They were synthesized ${ }^{6}$ following the method introduced by Ramsden ${ }^{23}$ i.e., by chemical reaction (leading to nucleation and growth) between an aqueous solution of $\mathrm{Cd}\left(\mathrm{ClO}_{4}\right)_{2}$ and HTe in the presence of thioglycolic acid (TGA), which forms a stabilizing layer on the surface of the particles, giving them a negative charge (the thiol group reacts with surface cadmium ions to form a monolayer shell of $\mathrm{CdS}$ around the $\mathrm{CdTe}^{6}$ ), followed by refluxing. Fig. 1 shows the optical absorption (recorded using a Perkin-Elmer Lambda 7 spectrophotometer) and photoluminescence (recorded using a Varian Cary Eclipse spectrophotometer with an excitation wavelength of $365 \mathrm{~nm}$ ) spectra of the received particles. Comparing our spectra to the extensive experimental data reported by $\mathrm{Yu}$ et al., ${ }^{32}$ we deduce that our particles have a mean diameter of about $3.25 \mathrm{~nm}$ and that their concentration $c_{\mathrm{b}}$ is $0.144 \mathrm{mg} / \mathrm{l}$.

Polycationic polydiallyldimethylammonium chloride (PDDA), $M_{\mathrm{r}} \sim 200$ 000-350 000 from Aldrich, was dissolved in distilled water to a concentration of $5 \mathrm{mg} / \mathrm{ml}$ before use.

The substrates were "monomode" planar optical waveguides purchased from MicroVacuum (Budapest), the high refractive index waveguiding layer being a pyrolysed sol-gel film of $\mathrm{Si}_{0.6} \mathrm{Ti}_{0.4} \mathrm{O}_{2}$ (Ref. 28) having a roughness of about $1 \AA$. The waveguides supported the zeroth order transverse magnetic (TM) and transverse electrical (TE) modes and had a grating coupler with an embossed surface corrugation (depth $\sim 5-10 \mathrm{~nm}$ and grating constant $=416.667 \mathrm{~nm}$ ). Prior to use, the waveguides were cleaned by placing them for $3 \mathrm{~min}$ in chromic acid at room temperature followed by a brief but thorough rinse in water, then placing them for $5 \mathrm{~s}$ in $2 \%$ aqueous potassium hydroxide solution, and finally rinsing them for $40 \mathrm{~min}$ in ultrapure water.

The waveguides were clamped to a miniature flowthrough cuvette made from PEEK and sealed with a Kalrez

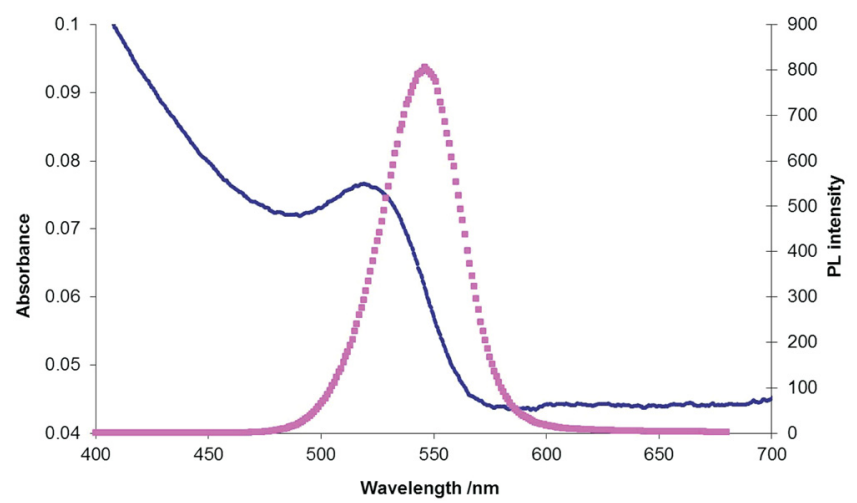

FIG. 1. The optical absorption (path length $1 \mathrm{~cm}$ ) and photoluminescence spectra of the CdTe particles as received (in aqueous suspension).
TABLE I. Process sequence of self-assembly. $\mathrm{c}$ a b a constitutes one deposition cycle.

\begin{tabular}{lc}
\hline \hline Label & Description $^{\mathrm{a}}$ \\
\hline $\mathrm{a}_{1}$ & Pure water to establish a baseline \\
$\mathrm{b}_{1}$ & PDDA solution for $20 \mathrm{~min}$ \\
$\mathrm{a}_{2}$ & Pure water for $2 \mathrm{~min}$ \\
$\mathrm{c}_{1}$ & CdTe nanoparticle suspension for $20 \mathrm{~min}$ \\
$\mathrm{a}_{3}$ & Pure water for $2 \mathrm{~min}$ \\
$\mathrm{~b}_{2}$ & PDDA solution for $20 \mathrm{~min}$ \\
$\mathrm{a}_{4}$ & Pure water for $2 \mathrm{~min}$ \\
$\mathrm{c}_{2}$ & Return to $\mathrm{c}_{1}$ to start a new cycle \\
\hline \hline
\end{tabular}

${ }^{\mathrm{a}}$ Liquids pumped at $1.67 \mu \mathrm{l} / \mathrm{s}$ using a peristaltic pump with Tygon tubing, corresponding to a wall shear rate of $5.9 \mathrm{~s}^{-1}$.

"o"-ring to the waveguide, which formed the floor of the cuvette (area $8 \times 2 \mathrm{~mm}$ ), and the assembly was mounted on the measuring head of a laboratory-built OWLS integrated optical scanner, ${ }^{10}$ with which the effective refractive indices $N$ of $\mathrm{TM}_{0}$ and $\mathrm{TE}_{0}$ were determined at $\lambda=632.816 \mathrm{~nm}$ (He-Ne laser) with a precision of $\sim 10^{-6}$. ${ }^{22}$ The sequence of solutions (Table I) passed through the cuvette was chosen to exactly match that of the previous work, ${ }^{2}$ and always ended with pure water. The temperature of the measurement and all solutions was $24.0^{\circ} \mathrm{C}$ and their refractive indices were close to that of pure water.

Fig. 2 shows typical results for a deposition run plotted as $N_{\mathrm{TM}}(t)$ and $N_{\mathrm{TE}}(t)$ vs $t$.

Simultaneously, solving the two 4-layer mode equations linking $N_{\mathrm{TM}}$ and $N_{\mathrm{TE}}$ to the optogeometric parameters of the deposited film yields the two unknown parameters of the assembled layer, i.e., its thickness $d_{\mathrm{A}}$ and refractive index $n_{\mathrm{A}},{ }^{9,29}$ assumed to be isotropic. These optogeometric parameters are plotted in Figure 3.

The total amount of matter deposited is quantifiable as the optical path $\delta$

$$
\delta=d_{\mathrm{A}}\left(n_{\mathrm{A}}-n_{\mathrm{C}}\right),
$$

which was calculated at the end of each cycle and plotted against the number of CdTe-PDDA deposition cycles ( $\mathrm{c} \mathrm{a} \mathrm{b}$ a) in Fig. 4. Growth is clearly linear as previously observed for APED involving only linear organic polymer polyelectrolytes. ${ }^{26}$ We can therefore exclude the "exponential" growth mechanism, ${ }^{16}$ which has been observed under certain conditions of APED. Similarly, plotting $d_{\mathrm{A}}$ vs layer number (not shown) yields a per-layer thickness of $3 \mathrm{~nm}$, which confirms the analysis of device cross-section carried out by Bertoni et al., ${ }^{2}$ and which corresponds to the mean diameter of the nanoparticles.

Initially (label $a_{1}$ ), a layer of PDDA is formed-it is the only macromolecular component in the system. The ability to repeat the CdTe-PDDA deposition sequence apparently at libitum amply demonstrates the operation of charge reversal upon each deposition. ${ }^{7}$ Nevertheless, the observed behaviour differs dramatically from the case in which polyelectrolytes only (i.e., no nanoparticles) are deposited (cf. Figure 5 in Ref. 26), in which the film thickness increases smoothly and monotonically during each deposition, and the film refractive index remains almost constant. The initial PDDA deposition 


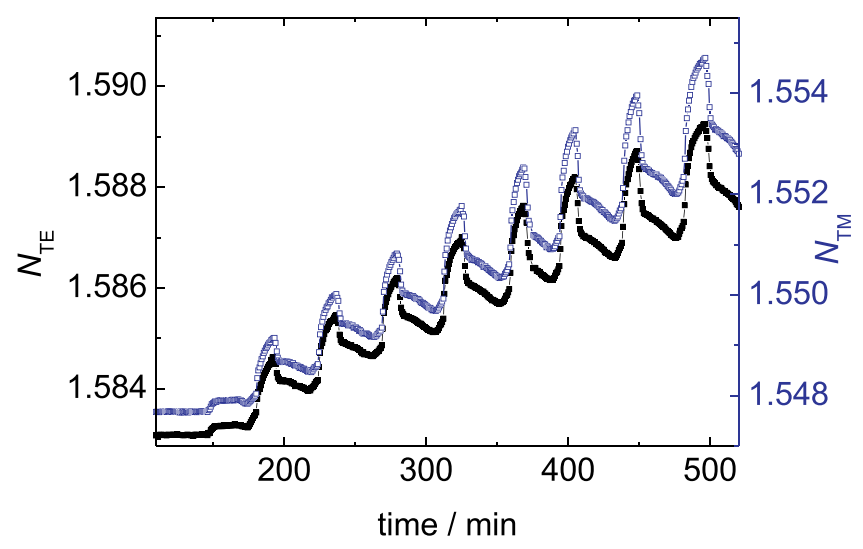

FIG. 2. Plots of $N_{\mathrm{TM}}(t)$ (open symbols, right hand scale) and $N_{\mathrm{TE}}(t)$ recorded during deposition of an initial PDDA layer on $\mathrm{Si}_{0.6} \mathrm{Ti}_{0.4} \mathrm{O}_{2}$ followed by 8 (CdTe, PDDA) alternating pairs.

and the first two depositions of CdTe are shown on an expanded timescale in Fig. 5, and decomposed into film thickness and refractive index in Figure 6. The expanded Figures 5 and 6 are highly revealing regarding the course of events.

Fig. 5 confirms that the polycationic PDDA (flow initiated at $b$ ) forms an initial layer on the negatively charged $\mathrm{Si}_{0.6} \mathrm{Ti}_{0.4} \mathrm{O}_{2},{ }^{25}$ as expected. ${ }^{26}$ The slight decrease when water flowed (arrow $\mathrm{a}_{2}$ ) is presumably due to the removal of weakly entangled polymer chains. The PDDA is expected to be adsorbed in loops, engendering a columnar structure with positive birefringence. ${ }^{7,26}$ Hence, the average refractive index of the first PDDA layer calculated by assuming an isotropic film severely underestimates the real value. ${ }^{9}$

When the CdTe nanoparticles flow over the substrate (starting at $\mathrm{c}_{1}$ ), film refractive index increases to a sharp peak, while the thickness of the film remains unchanged, and during the increase of $n_{\mathrm{A}}$, the total mass deposited (tracked as optical path or $N_{\mathrm{TM}}$ ) shows a small linear increase with time. From the absence of thickness change we deduce that the nanoparticles are entering small pores in the polymer polyelectrolyte layer, for the existence of which evidence has already been obtained. ${ }^{30}$ This process would naturally come to an end when the pores are filled. At that point (marked by the

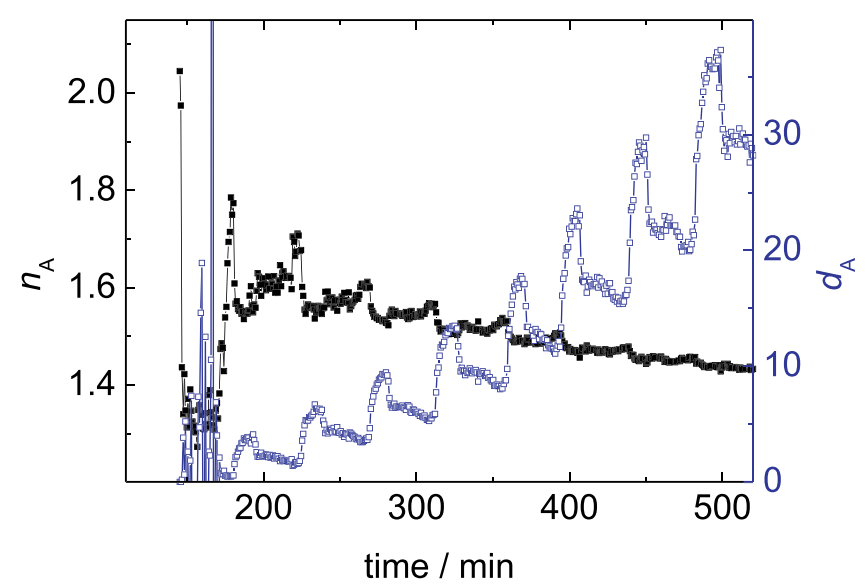

FIG. 3. Thickness $d_{\mathrm{A}}$ (empty symbols, scale on the right) and refractive index $n_{\mathrm{A}}$ (filled symbols, scale on the left), calculated from the data of Fig. 2 assuming no birefringence.

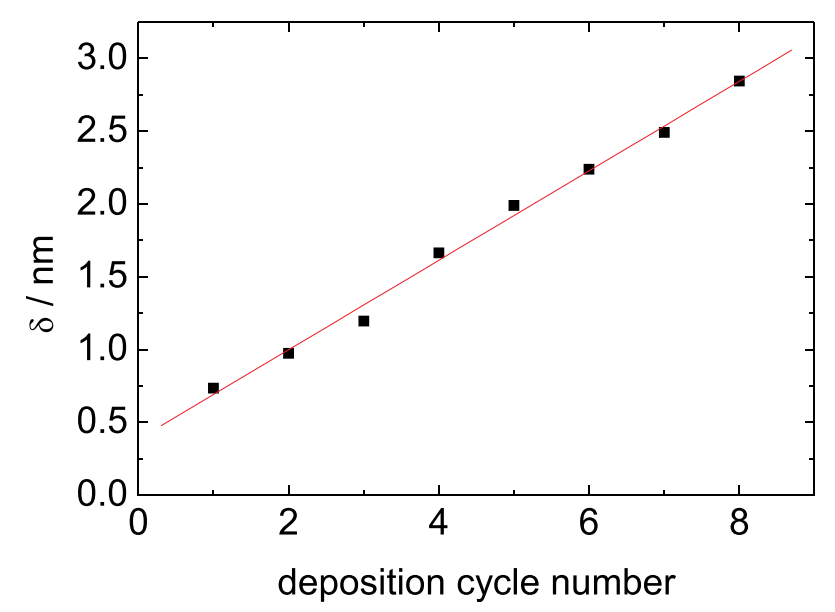

FIG. 4. The total amount of matter represented by the optical path $\delta$ (Eq. (1)) vs the number of (c a b a) deposition cycles (Table I).

arrow labeled $\gamma$ on Figs. 5 and 6), the thickness starts to increase, implying nanoparticle deposition onto, rather than within, the polymer (and eventually reaches a plateau, as expected when charge compensation has been achieved) and concomitantly film refractive index decreases; $\gamma$ also marks the abrupt change of gradient of the variation of $N_{\mathrm{TM}}$ (tracking total mass deposited) with time. Total mass deposited, film thickness, and film refractive index each reach a new plateau, the refractive index reaches it well before the other two variables. We have previously shown ${ }^{9}$ that solving the mode equations to yield film thickness and average refractive index with the assumption that the film is optically isotropic decreases the apparent average refractive index if the film is, in fact, anisotropic with Wiener's form factor $u \rightarrow 0$, corresponding to a columnar structure with positive birefringence. Hence, the pore-filling diminishes the columnar nature of the film.

After the plateaus are reached, subsequently flowing water (starting at $\mathrm{a}_{3}$ ) over the film rapidly removes a substantial fraction (about one third) of the deposit. From the magnitude of the removal, it can be deduced that nanoparticles are being removed. If at least some of them are reversibly adsorbed, replacing the nanoparticle solution with pure

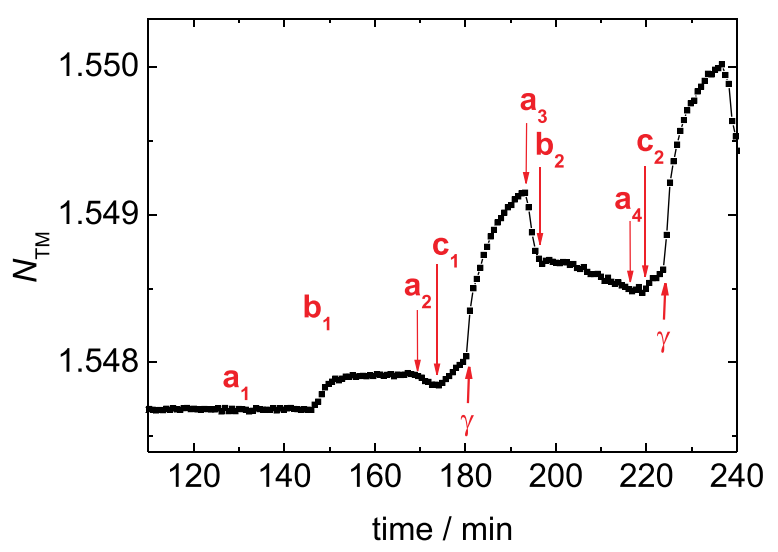

FIG. 5. The deposition of an initial PDDA layer on $\mathrm{Si}_{0.6} \mathrm{Ti}_{0.4} \mathrm{O}_{2}$ followed by the first two (CdTe,PDDA) alternating pairs (enlargement of the first part of Fig. 2), tracked as $N_{\mathrm{TM}}$. The key to the Roman labels is in Table I; $\gamma$ indicates the abrupt change of rate of mass deposition during exposure to the nanoparticles. 


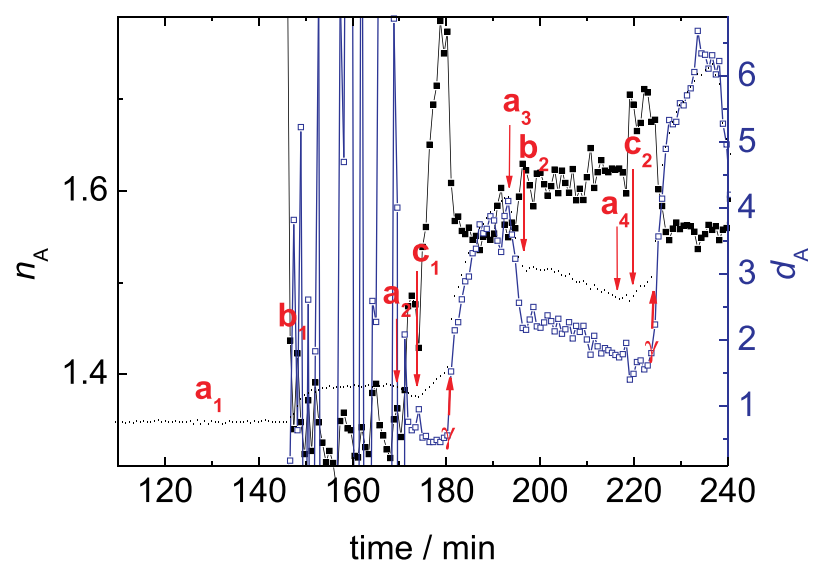

FIG. 6. The deposition of an initial PDDA layer on $\mathrm{Si}_{0.6} \mathrm{Ti}_{0.4} \mathrm{O}_{2}$ followed by the first two (CdTe, PDDA) alternating pairs (enlargement of the first part of Fig. 3). The key to the Roman labels is in Table I; $\gamma$ indicates the abrupt change of rate of mass deposition during exposure to the nanoparticles (cf. Fig. 5). No great significance should be attached to the large fluctuations of $n_{\mathrm{A}}$ and $d_{\mathrm{A}}$ at the beginning of deposition, prior to arrow $\mathrm{a}_{2}$. The deposited layer is ill defined optically and its refractive index is close to that of the covering medium $n_{\mathrm{C}}$. The mode equations linking the $N$ with $n_{\mathrm{A}}$ and $d_{\mathrm{A}}$ contain difference terms $n_{\mathrm{A}}-n_{\mathrm{C}}$ as denominators, which abruptly become very large as the difference tends to zero. Furthermore, second harmonic generation experiments (for APED with polymer polyelectrolytes only) have shown that considerable restructuring occurs within the first layer as it becomes established. ${ }^{18}$

water will perturb the equilibrium and the system will relax to a new state with a smaller number of adsorbed nanoparticles. The hydrodynamic boundary layer thickness $\delta_{\mathrm{h}}$ depends on the particle diffusivity $D$, the wall shear rate and a cell constant; ${ }^{24}$ using the Stokes-Einstein relation to compute $D\left(1.56 \times 10^{-6} \mathrm{~cm}^{2} / \mathrm{s}\right)$, we obtain $\delta_{\mathrm{h}}=175 \mu \mathrm{m}$, from which we can safely exclude the possibility that flowinduced mechanical shear is contributing to particle removal. The removal is accompanied by significant densification of the film, as evinced by the increase in average refractive index $n_{\mathrm{A}}$, implying that the adsorbed particles form a quasilayer on the periphery of the film proper.

The flow of polymer polyelectrolyte (starting at $b_{2}$ ) causes no further change in film refractive index, accompanied by a slight diminution of the thickness (and concomitant diminution of the deposited mass), which rules out further densification of the film as the interpretation of the data. The ability of mobile nanoparticles to scavenge organic films of surfactant has been previously demonstrated, ${ }^{20}$ and this phenomenon offers a possible explanation of the diminution of thickness and mass: mobile polyelectrolyte molecules are abstracting some of the CdTe nanoparticles and removing them from the film.

Finally, during the water flow (starting at $\mathrm{a}_{4}$ ) following polymer deposition, mass, refractive index, and thickness are all unchanged, demonstrating film stability. The sequence (c, $\gamma, \mathrm{a}, \mathrm{b}, \mathrm{a})$ was repeatable essentially ad libitum with qualitatively the same occurrences but the magnitude of the changes corresponding to the feature labelled $\gamma$ steadily increased (total deposited mass and thickness) or decreased (average refractive index) with successive cycles (Figs. 2 and 3). The trend of $n_{\mathrm{A}}$ merely indicates that the structural changes during deposition take place in the surface layer only, hence, the overall effect (averaged over all layers) diminishes as the number of layers increases. The increase of transient peak thickness and total deposited mass may be due to an increasingly rough surface (cf. Ref. 1).

High-resolution transmission electron microscopy after 5 deposition cycles shows a uniform dense packing (Fig. 1 in Ref. 2) consistent with the X-ray reflectivity-based estimate of an average film density of $2.3-2.6 \mathrm{~g} / \mathrm{cm}^{3}$, , corresponding to a filling factor of 0.27 (the exact conditions of the X-ray reflectivity measurements were not specified but we assume that the degree of hydration was similar to that in our experiments). This must correspond to the final average layer refractive index, which converges to a value of about 1.45 (Fig. 3). According to the "parallel" effective medium approximation

$$
n_{\mathrm{A}}=\theta n_{\mathrm{CdTe}}+\theta_{\text {polymer }} n_{\text {polymer }}+\theta_{\text {polymer }} \theta_{\text {water }} n_{\text {water }}
$$

where $n_{\mathrm{CdTe}}$ is the refractive index of bulk CdTe $(=2.67$ at $633 \mathrm{~nm}$ (Ref. 8)), $n_{\text {polymer }}$ can be estimated as 1.55 from data for a similar polymer, ${ }^{12}$ and $n_{\text {water }}=1.33$. $\theta_{\text {water }}$ is the fractional hydration of a pure polyelectrolyte layer (i.e., in the absence of nanoparticles). It is not known very precisely; we take 0.25 as a reasonable estimate. ${ }^{27}$ Hence, from $1=\theta_{\mathrm{CdTe}}$ $+\theta_{\text {polymer }}+\theta_{\text {polymer }} \theta_{\text {water, we have }} \theta_{\text {polymer }}=0.58$. From Eq. (2), we therefore obtain $n_{\mathrm{A}}=1.81$. This is indeed the peak refractive index reached at $\gamma$ (Fig. 6). The following rapid decrease of apparent average refractive index while the strongly refracting $\mathrm{CdTe}$ particles continue to be added to the system makes no sense unless $n_{\mathrm{A}}$ is being progressively underestimated due to increasingly positive birefringence, ${ }^{9,15}$ which, in turn, is due to a predominantly columnar molecular orientation, which has been established as a characteristic feature of polymer polyelectrolyte-only APED. ${ }^{26}$

In conclusion, OWLS reveals itself as a very useful in situ technique for yielding fine kinetic structural information about the deposition, well satisfying the need for critical scrutiny of the assembly process. Careful analysis of this information revealed that the addition of the nanoparticles to the polymer polyelectrolyte is a two-stage process: initially, the particles enter nanopores in the polymer layer and this stage strictly precedes the formation of a nanoparticle-rich adlayer. The rate of the former process is likely to be much slower than the latter. Using the known filling factor to calibrate the optical path in terms of numbers of CdTe particles, the slow, uniform rate of pore-filling is about $5 \times 10^{9}$ particles $\mathrm{cm}^{-2}$ $\mathrm{s}^{-1}$ (and tends to make the film more isotropic), and the adlayer formation takes place initially about twentyfold faster but the rate rapidly diminishes to zero. For comparison, the diffusion-limited flux of particles to the surface in the absence of long-range attractive (electrostatic) forces is $c_{\mathrm{b}} D / \delta_{\mathrm{h}} \approx 10^{8}$ particles $\mathrm{cm}^{-2} \mathrm{~s}^{-1}$. What is still not clear is why there is such an abrupt transition between the two régimes.

In strong contrast to the alternating deposition of organic polycation and -anion, the alternating deposition of an organic polycation and polyanionic CdTe nanocrystals does not proceed as a simple sequential buildup of layers of the two substances, although the net deposited mass still increases linearly with the number of deposition cycles.

The scope of these findings goes well beyond the fabrication of electroluminescent devices that originally inspired this work; they need to be taken into account whenever an 
industrial fabrication process based on APED ("layer-bylayer") involving nano-objects and polymers is being contemplated.

We thank Nikolai Gaponik, TU Dresden, for the gift of CdTe nanoparticles and Henrik Pedersen, Technical University of Denmark, for the loan of optical and electronic components. R.H. acknowledges the award of a Marie Curie (EIF) fellowship by the European Commission and support from the Lendület Programme of the Hungarian Academy of Sciences.

${ }^{1}$ F. Bentrem, R. B. Pandey, and F. Family, "Roughening, deroughening, and nonuniversal scaling of the interface width in electrophoretic deposition of polymer chains," Phys. Rev. E 62, 914-917 (2000).

${ }^{2}$ C. Bertoni, D. Gallardo, S. Dunn, N. Gaponik, and A. Eychmuller, "Fabrication and characterization of red-emitting electroluminescent devices based on thiol-stabilized semiconductor nanocrystals," Appl. Phys. Lett. 90, 034107 (2007).

${ }^{3}$ T. Boudou, T. Crouzier, K. Ren, G. Blin, and C. Picart, "Multiple functionalities of polyelectrolyte multilayer films: new biomedical applications," Adv. Mater. 22, 441-467 (2010).

${ }^{4}$ Multilayer Thin Films, edited by G. Decher and J. B. Schlenoff (Wiley-VCH, Weinheim, 2003).

${ }^{5}$ G. Decher, J.-D. Hong, and J. Schmitt, "Buildup of ultrathin multilayer films by a self-assembly process: III. Consecutively alternating adsorption of ionic and cationic polyelectrolytes on charged surfaces," Thin Solid Films 210/211, 831-835 (1992).

${ }^{6}$ N. Gaponik, D. V. Talapin, A. L. Rogach, K. Hoppe, E. V. Shevchenko, A. Kornowski, A. Eychmu1ller, and H. Weller, "Thiol-capping of CdTe nanocrystals: An alternative to organometallic synthetic routes," J. Phys. Chem. B 106, 7177-7185 (2002).

${ }^{7}$ A. Yu. Grosberg, T. T. Nguyen, and B. I. Shklovskii, "The physics of charge inversion in chemical and biological systems," Rev. Mod. Phys. 74, 329-345 (2002).

${ }^{8}$ P. Hlidek, J. Bok, J. Franc, and R. Grill, "Refractive index of CdTe: Spectral and temperature dependence," J. Appl. Phys. 90, 1672-1674 (2001).

${ }^{9} \mathrm{R}$. Horvath and J. J. Ramsden, "Quasi-isotropic analysis of anisotropic thin films on optical waveguides," Langmuir 23, 9330-9334 (2007).

${ }^{10}$ R. Horvath, H. C. Pedersen, and F. J. G. Cuisiner, "Guided wave sensing of polyelectrolyte multilayers," Appl. Phys. Lett. 88, 111102 (2006).

${ }^{11}$ R. K. Iler, "Multilayers of colloidal particles," J. Colloid Interface Sci. 21, 569-594 (1966).

${ }^{12}$ J.-H. Kim, J.-H. Hwang, and T.-Y. Lim, “A layer-by-layer self-assembly method for organic-inorganic hybrid multilayer thin films," J. Ceram. Process. Res. 10, 770-773 (2009).

${ }^{13}$ D. S. Kommireddy, A. A. Patel, T. G. Shutava, D. K. Mills, and Yu. M. Lvov, "Layer-by-layer assembly of $\mathrm{TiO}_{2}$ nanoparticles for stable hydrophilic biocompatible coatings," J. Nanosci. Nanotechnol. 5, 1081-1087 (2005).

${ }^{14}$ N. A. Kotov, I. Dékány, and J. H. Fendler, "Layer-by-layer self-assembly of polyelectrolyte-semiconductor nanoparticle composite films," J. Phys. Chem. 99, 13065-13069 (1995).
${ }^{15}$ N. Kovacs, D. Patko, N. Orgovan, S. Kurunczi, J. J. Ramsden, F. Vonderviszt, and R. Horvath, "Optical anisotropy of flagellin layers: In situ and label-free measurement of adsorbed protein orientation using OWLS," Anal. Chem. 85, 5382-5389 (2013).

${ }^{16}$ P. Lavalle, C. Picart, J. Mutterer, Cs. Gergely, H. Reiss, J.-C. Voegel, B. Senger, and P. Schaaf, "Modelling the buildup of polyelectrolyte multilayer films having exponential growth," J. Phys. Chem. B 108, 635-648 (2004).

${ }^{17}$ Yu. Lvov, K. Ariga, I. Ichinose, and T. Kunitake, "Molecular film assembly via layer-by-layer adsorption of oppositely charged macromolecules (linear polymer, protein and clay) and concanavalin A and glycogen," Thin Solid Films 284-285, 797-801 (1996).

${ }^{18} \mathrm{R}$. McAloney and M. C. Goh, "In situ investigations of polyelectrolyte film formation by second harmonic generation," J. Phys. Chem. B 103, 10729-10732 (1999).

${ }^{19}$ S. A. McClure, B. J. Worfolk, D. A. Rider, R. T. Tucker, J. A. M. Fordyce, M. D. Fleischauer, K. D. Harris, M. J. Brett, and J. M. Buriak, "Electrostatic layer-by-layer assembly of CdSe nanorod/polymer nanocomposite thin films," Appl. Mater. Interfaces 2, 219-229 (2010).

${ }^{20} \mathrm{M}$. Máté and J. J. Ramsden, "Colloidal particles efficiently scavenge fatty acid Langmuir-Blodgett films," J. Dispersion Sci. Technol. 19, 875-883 (1998).

${ }^{21}$ M. Máté and J. J. Ramsden, “Addition of particles of alternating charge," J. Chem. Soc., Faraday Trans. 94, 2813-2816 (1998).

${ }^{22}$ N. Orgovan, D. Patko, Cs. Hos, S. Kurunczi, B. Szabo, J. J. Ramsden, and R. Horvath, "Sample handling in surface sensitive chemical and biological sensing: A practical review of basic fluidics and analyte transport," Adv. Colloid Interface Sci. 211, 1-16 (2014).

${ }^{23}$ J. J. Ramsden, "The nucleation and growth of small CdS aggregates by chemical reaction," Surf. Sci. 156, 1027-1039 (1985).

${ }^{24}$ J. J. Ramsden, "Review of new experimental methods for investigating random sequential adsorption," J. Stat. Phys. 73, 853-877 (1993).

${ }^{25}$ J. J. Ramsden and M. Máté, "Kinetics of monolayer particle deposition," J. Chem. Soc., Faraday Trans. 94, 783-788 (1998).

${ }^{26}$ J. J. Ramsden, Yu. M. Lvov, and G. Decher, " Optical and x-ray structural monitoring of molecular films assembled via alternate polyion adsorption," Thin Solid Films 254, 246-251 (1995); 261, 343-344 (1995).

${ }^{27}$ M. Schönhoff, V. Ball, A. R. Bausch, C. Dejugnat, N. Delorme, K. Glinel, R. von Klitzing, and R. Steitz, "Hydration and internal properties of polyelectrolyte multilayers," Colloids Surf. A 303, 14-29 (2007).

${ }^{28}$ I. Szendro", "Art and practice to emboss gratings onto sol-gel waveguides," Proc. SPIE 4284, 80-87 (2001).

${ }^{29} \mathrm{~K}$. Tiefenthaler and W. Lukosz, "Sensitivity of grating couplers as integrated-optical chemical sensors," J. Opt. Soc. Am. B 6, 209-220 (1989).

${ }^{30} \mathrm{~F}$. Vaca Chavez and M. Schönhoff, "Poor size distributions in polyelectrolyte multilayers determined by cryoporometry," J. Chem. Phys. 126, 104705 (2007).

${ }^{31}$ Q. Xing, S. R. Eadula, and Yu. M. Lvov, "Cellulose fiber-enzyme composites fabricated through layer-by-layer nanoassembly," Biomacromolecules 8, 1987-1991 (2007).

${ }^{32}$ W. W. Yu, L. Qu, W. L. Guo, and X. Peng, "Experimental determination of the extinction coefficient of CdTe, CdSe and CdS nanocrystals," Chem. Mater. 15, 2854-2860 (2003).

${ }^{33}$ Y. Zhang and J. R. G. Evans, "Approaches to the manufacture of layered nanocomposites,” Appl. Surf. Sci. 258, 2098-2102 (2012).

${ }^{34}$ Z. Zheng, J. McDonald, T. Shutava, G. Grozdits, and Yu. Lvov, "Layerby-layer nanocoating of lignocellulose fibers for enhanced paper properties," J. Nanosci. Nanotechnol. 6, 624-632 (2006). 